\title{
A História do TDAH - Evolução
}

The History of ADHD - Evolution

\section{La Historia del TDAH - Evolución}

\author{
Aline dos Santos Moreira de Carvalho \\ ORCID: https://orcid.org/0000-0001-9965-9566 \\ Universidade Columbia del Paraguay, Paraguai \\ E-mail: bioaline2017@yahoo.com \\ Liliane Musumeci Ferreira \\ ORCID: https://orcid.org/0000-0001-8749-0652 \\ Universidade Columbia del Paraguay, Paraguai \\ E-mail: lilianemusumeciferreira@gmail.com \\ Léia Flauzina da Silva Albuquerque \\ ORCID: https://orcid.org/ 0000 -002-6942-1116 \\ Universidade Columbia del Paraguay, Paraguai \\ E-mail: psicopedagogarjleiaflauzina@gmail.com \\ Lucélia Aparecida Jagobucci \\ ORCID: https://orcid.org/0000-0001-7517-4441 \\ Universidade Columbia del Paraguay, Paraguai \\ E-mail: jagobucci@gmail.com \\ Karla de Lourdes Antunes Souza \\ ORCID: https://orcid.org/0000-0002-2611-9611 \\ Universidade Columbia del Paraguay, Paraguai \\ E-mail: kaka.antunes@hotmail.com \\ Jean Carlos Triches \\ ORCID: https://orcid.org/0000-0002-7127-0193 \\ Universidade Comunitária da Região de Chapecó, Brasil \\ Faculdade do Oeste de Santa Catarina, Brasil \\ E-mail: jean.triches@gmail.com \\ Luciana Menezes de Almeida \\ ORCID: https://orcid.org/0000-0002-5854-1956 \\ Universidade Columbia del Paraguay, Paraguai \\ E-mail: profpsilu@gmail.com \\ Ingrid dos Santos Pereira \\ ORCID: https://orcid.org/0000-0002-2995-2385 \\ Universidade Columbia del Paraguay, Paraguai \\ E-mail: isp.pedagoga@gmail.com \\ Erika dos Santos Pereira \\ ORCID: https://orcid.org/0000-0002-2995-2385 \\ Universidade Columbia del Paraguay, Paraguai \\ E-mail: espereira1337@gmail.com \\ Pacifico Ferraz Souto \\ ORCID: https://orcid.org/0000-0001-9639-665X \\ Universidade Columbia del Paraguay, Paraguai \\ E-mail: Pacifico.souto@educacao.mg.gov.br
}

\section{Resumo}

O Transtorno de Déficit de Atenção e Hiperatividade apresenta em sua história e descrição complexidades de significados, conceitos e algumas controvérsias. I consenso sobre a descrição ocorre de forma geral, porém sob o aspecto neuro-psiquiátrico, alguns cientistas apresentam discordâncias, o que influenciou a história do transtorno assim como seu processo evolutivo como patologia. Nessa perspectiva, o presente estudo propõe descrever a história do transtorno pontuando algumas especificidades e contradições. Para tal, a metodologia utilizada neste estudo é de caráter qualitativo, em relação aos objetivos, é uma pesquisa exploratória e descritiva e referente aos procedimentos de pesquisa, o estudo caracterizou-se como bibliográfico. A pesquisa foi realizada em meio virtual, em bases de dados confiáveis como Scielo, com artigos científicos e livros em formato pdf, com os descritores TDAH, história e evolução, que gerou resultados e discussão divididos em três tópicos: considerações gerais, contexto histórico e evolutivo e algumas notas. As considerações finais compreendem as impressões e conclusões da autora.

Palavras-chave: Evolução; História; TDAH. 


\begin{abstract}
Attention Deficit Hyperactivity Disorder presents in its history and description complexities of meanings, concepts and some controversies. I consensus on the description occurs in general, but from the neuro-psychiatric point of view, some scientists have disagreements, which influenced the history of the disorder as well as its evolutionary process as a pathology. In this perspective, the present study proposes to describe the history of the disorder, pointing out some specificities and contradictions. To this end, the methodology used in this study is qualitative, in relation to the objectives, it is an exploratory and descriptive research and referring to the research procedures, the study was characterized as bibliographic. The research was carried out in a virtual environment, in reliable databases such as Scielo, with scientific articles and books in pdf format, with the descriptors ADHD, history and evolution, which generated results and discussion divided into three topics: general considerations, historical context and evolution and some notes. The final considerations comprise the author's impressions and conclusions.
\end{abstract}

Keywords: Evolution; History; ADHD.

\title{
Resumen
}

El Trastorno por Déficit de Atención con Hiperactividad presenta en su historia y descripción significados complejos, conceptos y algunas controversias. El consenso sobre la descripción se da en general, pero bajo el aspecto neuropsiquiátrico, algunos científicos presentan desacuerdos, que influyeron tanto en la historia del trastorno como en su proceso evolutivo como patología. Desde esta perspectiva, este estudio se propone describir la historia del trastorno, señalando algunas especificidades y contradicciones. Para tal, la metodología utilizada en este estudio es cualitativa, en relación a los objetivos, se trata de una investigación exploratoria y descriptiva y en referencia a los procedimientos de investigación, el estudio se caracterizó como bibliográfico. La investigación se realizó en un entorno virtual, en bases de datos confiables como Scielo, con artículos científicos y libros en formato pdf, con los descriptores TDAH, historia y evolución, lo que generó resultados y discusión divididos en tres temas: consideraciones generales, contexto histórico y rodando y algunas notas. Las consideraciones finales incluyen las impresiones y conclusiones del autor.

Palabras clave: Evolución; Historia; TDAH.

\section{Introdução}

O Transtorno de Déficit de Atenção e Hiperatividade (TDAH) apresenta, no aspecto descritivo e histórico complexidades de conceitos e significados assim como controvérsias.

Em linhas gerais, existe um razoável consenso que apresenta o TDAH como um transtorno do desenvolvimento de fundamento genético e neuroquímico, que se apresenta normalmente em crianças de idade pré-escolar que pode persistir até a fase adulta, sendo caracterizado por sintomas como agitação, impulsividade, dificuldade de manter a atenção, desatenção (Bukley, 2002 apud Pereira, 2009). Porém, em relação ao contexto neuro-psiquiátrico, existem controvérsias entre alguns cientistas sobre o assunto.

Sendo assim, a história do TDAH, referida como oficial, evolui juntamente com as pesquisas e estudos que modificam a descrição do transtorno, por vezes alterando sua classificação e enquadrando sintomas diversos, assim como alternando a prevalência de outros, como o caso da desatenção, hiperatividade ou a combinação de ambos.

Nessa perspectiva, o presente estudo propõe descrever a história do transtorno pontuando algumas especificidades e contradições.

Para tal, a metodologia utilizada neste estudo é de caráter qualitativo pois tal abordagem oferece e possibilita uma visão para [...] o universo dos significados, dos motivos, das aspirações, das crenças, dos valores e das atitudes." (Minayo et al., 2009, p. 21).

Em relação aos objetivos, é uma pesquisa exploratória e descritiva (GIL, 2008) pois busca explorar conceitos, significados e classificações do TDAH durante sua história e evolução.

No que concerne os procedimentos de pesquisa, o estudo caracterizou-se como bibliográfico que, segundo Gil (2008), é desenvolvida com base em material já elaborado, principalmente em artigos científicos e livros. A pesquisa foi realizada em meio virtual, em bases de dados confiáveis como Scielo, com artigos científicos e livros em formato PDF com os descritores TDAH, história e evolução. 


\section{Considerações Gerais}

Pereira (2009) afirma que a nomenclatura TDAH surgiu em 1994 com a publicação da versão Diagnostic Statistical Manual of Mental Disorder's IV (DSM IV), editado pela American Psychiatric Association, sendo expandido dentro e fora dos contextos psiquiátricos e considerado "o transtorno mais comum em crianças e adolescentes encaminhados para serviços especializados" (Abda, 2008e apud Pereira, 2009).

Segundo a mesma, Latour (2000) propõe abrir horizontes (que chama de caixas-pretas) para que seja realizada uma investigação sociológica de conceitos científicos já aceitos pela sociedade e eu sejam estáveis e comprovados. Assim, insere a possibilidade de estudos sobre o diagnóstico psiquiátrico do TDAH bem como o levantamento de questões sobre a legitimização dessa patologia, uma vez que está sendo debatida, comentada, referida e produzida (Pereira, 2009).

O Transtorno de Déficit de Atenção e Hiperatividade e seus desdobramentos no meio científico e educacional é uma questão que atravessa inúmeras controvérsias, discursos e materialidades e que está em evidência em discussões e debates entre os séculos XX e XXI (observando o fato de que nessa época existe a problematização da ciência enquanto saber imparcial, objetivo e generalizável (Ferreira \& Moscheta, 2019).

Para Ferreira e Moscheta (2019), construir uma realidade acerca do TDAH implica considerar que o TDAH não é único, não existe uma única descrição assertiva sobre o transtorno capaz de descrevê-lo completamente, mas sim múltiplas versões relacionadas ou não, pois para desenvolver uma realidade acerca daquele, faz-se necessário mobilizar política, discursos, laboratórios, instituições, textos científicos (Moraes, 2001/2002 apud Ferreira \& Moscheta, 2019).

De acordo com Hacking (2007 apud Brzozoowskil \& Caponi, 2009), um filósofo da ciência, habitamos num mundo de classificações e que possuem efeitos particulares quando referidas a comportamentos pessoais. Dessa forma, são gerados tipos de pessoas, formados pelas que compartilham do mesmo problema, e as instituições são responsáveis por confirmar a existência desses tipos de pessoas e afazer sua legitimização através do conhecimento possuído pelos especialistas (os primeiros a classificar).

Para Vasconcelos (2003 apud Brzozoowskil \& Caponi, 2009) o TDAH é o diagnóstico psiquiátrico mais comum da infância, caracterizado por três sintomas principais que são a hiperatividade, a desatenção e a impulsividade, que possui uma taxa média de prevalência, no Brasil, de 3,6 a $5 \%$ da população escolar e, em outros estudos, sobe para $12 \%$ a prevalência, sendo seu diagnóstico realizado através de critérios estabelecidos em guias oficiais e fundamentalmente clínico e subjetivo.

Nesse contexto complexo de conceitos, significados e classificações, o TDAH permanece como um dos transtornos mais referidos, debatidos e discutidos no meio acadêmico educacional, atualmente.

\section{Contexto Histórico e Evolutivo}

Caliman (2010) discorre sobre a problemática existente com relação ao diagnóstico do TDAH, que influencia diretamente em sua história enquanto transtorno psiquiátrico que seria expressa na pergunta sobre as descrições desse transtorno. Segundo a mesma, uma vez que não seja necessária a presença dos três sintomas (desatenção, hiperatividade e impulsividade), a história desse transtorno pode ser orientada pela predominância de um dos três sintomas centrais do mesmo.

Considerando que para a medicina os três sintomas relacionam-se, a história do TDAH se desenrola enquanto os três sintomas alternam no lugar de maior ou menor importância, porém os autores que estudam o transtorno concordam que os sintomas manifestam-se no ambiente escolar, trazendo esse para a história daquele (Caliman, 2010).

Na história oficial da desordem, o ambiente escolar se faz presente nos quatro precursores do TDAH. Segundo o discurso crítico, no campo infantil, a história do TDAH deve ser analisada sob os aspectos de controle e medicalização infantil (Schrag \& Divoky, 1975; Werner, 2001), poder da instituição sobre a criança indisciplinada (Conrad, 1975, 1976), discurso médico sobre a criança idiota e do imbecil moral (segunda metade do século XIX) (Rafalovich, 2002), construção da criança hiperativa 
comparada a eletricidade (segunda metade do século XIX) (Barbetti, 2003) e a criança hiperativa que é inapta e instável (século XIX) (Dupanloup, 2004) (apud Caliman, 2010).

Para o discurso adolescente, deve-se considerar a prática médica em adolescentes delinquentes e desviantes. Para o adulto, história seria desde 1980. Existem autores que relacionam a história do transtorno com o desenvolvimento de medicações, indústria farmacêutica e comércio assim como outros relacionam o TDAH ao desenvolvimento de tecnologias de imagem cerebral e neurologia; outros ainda, cientistas sociais, relacionam a história do TDAH com a evolução tecnológica, excesso de informação, velocidade de informação, perda da autoridade da família, identidades descartáveis, o que os leva a problematizar a existência ou não patológica do TDAH (Caliman, 2010).

De acordo com a Cartilha PROIS, as primeiras descrições de crianças que apresentavam comportamento semelhante as que hoje são conhecidas como TDAH surgiram na literatura infantil alemã, na metade do século XIX, tais livros foram publicados no Brasil em 1950 e descreviam crianças muito levadas que tinham dificuldades em obedecer às regras impostas pelos pais (Prois, 2021).

Em 1917, Von Economo, médico, descreveu pela primeira vez a patologia hoje conhecida como TDAH:

Temos nos deparado com uma série de casos nas instituições psiquiátricas que não fecham com nenhum diagnóstico conhecido. Apesar disso, eles apresentam similaridades quanto ao tipo de início do quadro e sintomatologia que nos força a agrupá-los em uma nova categoria diagnóstica... Estas crianças parecem ter perdido a inibição, tornam-se inoportunas, impertinentes e desrespeitosas. São cheias de espertezas, muito falantes... (Economo, 1917 apud Prois, 2021, p. 9).

De acordo com a mesma Cartilha, o TDAH, ao longo da história recebeu várias denominações, dentre essas, lesão cerebral mínima, síndrome hipercinética, disfunção cerebral mínima, considerando que cada nova denominação representava um avanço científico em descobertas sobre o transtorno; o diagnóstico é clínico, porém não existe nenhum tipo de exame nem laboratorial nem de imagem que possa conduzir a um diagnóstico sozinho pois, muitas vezes, deve ser feito por uma equipe multidisciplinar através de uma abordagem com muitas avaliações (PROIS, 2021).

De acordo com Caliman (2010) existem algumas histórias sobre o TDAH, porém julga-se a oficial a que compreende que a criança com TDAH surgiu na medicina na metade do século XIX, foi a que possuía defeito de controle moral, portadora de deficiência mental leve ou branda, afetada por encefalite letárgica, hiperativa, hipercinética, com cérebro moderadamente disfuncional, com déficit de atenção e finalmente portadora de déficit de atenção e hiperatividade. Tal versão é conhecida como oficial e dominante pois dentre os debates científico, político, popular e econômico, é conhecida como a história dos diagnósticos de TDAH (Caliman, 2010).

Rezende (2016) destaca algumas etapas cronológicas da evolução da patologia, de forma generalizada pera melhores esclarecimentos do público leigo. O autor divide a história do TDAH em algumas partes, somando 200 anos dessa:

I - Século XVIII, doenças da atenção: Alexander Crichton (1763 - 1856) foi um médico escocês conhecido como o primeiro autor a descrever as características do transtorno, como uma desatenção patológica;

II - Heinrich Hoffman (1809-1894), psiquiatra alemão, faz algumas descrições em livros por ele publicados, como "Felipe, o inquieto". Nas obras, as crianças são inquietas, facilmente distraídas e agressivas;

III - George Frederic Still (1868-1941), pediatra britânico, apresentou uma conferência onde afirmou que em alguns casos, as condições psíquicas estavam relacionadas ao defeito de controle moral das crianças (sem retardo mental ou qualquer problema físico). Descreveu algumas crianças como: impulsivas, imediatistas, e não capazes de sustentar a atenção (relatados por pais e professores);

IV - No início do século XX, muitos médicos relacionaram danos causados no cérebro com problemas de comportamento, que foi confirmada durante a epidemia de encefalite aguda (1915 - 1930); 
V - Em 1932, Franz Kramer (1878 - 1967) e Hans Pollnow (1902 -1943) publicaram estudos sobre a doença hipercinética da infância, transtorno com acentuada inquietação motora. Esses médicos enfatizaram mais a impulsividade e a agitação do que os aspectos morais e apesar de crerem ser de característica infantil, poderia permanecer na vida adulta;

VI - Em 1937, o psiquiatra americano Charles Bradley (1902 - 1979) descobriu um medicamento que possuía efeitos positivos em crianças com problemas de comportamento;

VII - Em 1954, a Ritalina aparece como principal medicamento para o tratamento de crianças hiperativas;

VIII - Em 1940 é introduzido o novo conceito de lesão cerebral mínima, que se baseava na ideia de que uma lesão mínima no cérebro ocasionaria alguns comportamentos hiperativos e alguns transtornos, em diferentes graus de severidade;

IX - Na década de 1960, foram apresentadas críticas ao conceito de lesão cerebral mínima, introduzindo o conceito de disfunção cerebral mínima, englobando outros transtornos;

X - Em 1968, a descrição do transtorno passou a ser incluída na $2^{a}$ edição do Manual Diagnóstico e Estatístico de Transtornos Mentais, intitulado de reação hipercinética da infância, que foi definida como Excesso de atividade, inquietação, distração e falta de atenção;

XI - Em 1970, o foco foi para a ênfase de déficit de atenção, com a publicação do III DSM, o transtorno foi renomeado para Transtorno de Déficit de Atenção (TDA), que poderia ocorrer com ou sem hiperatividade;

XII - No início da década de 80, para melhorar a conceituação e critérios, o transtorno foi renomeado para Transtorno de Déficit de Atenção e Hiperatividade;

XIII - Na década de 1990, já haviam muitos estudos sobre as bases neurológicas do TDAH, entre os quais que o transtorno não é uma condição da infância, mas persiste na maioridade; e que existem três subtipos: predominantemente desatento, predominantemente hiperativo-impulsivo e os dois combinados com os sintomas dos anteriores;

XIV - A DSM-V utiliza quase que as mesmas definições, nomes e critérios para o diagnóstico que o DSM - IV, utilizando de um padrão persistente de desatenção que possui dezoito sintomas diferentes (Rezende, 2016).

Figura 1 - Linha do tempo do TDAH.

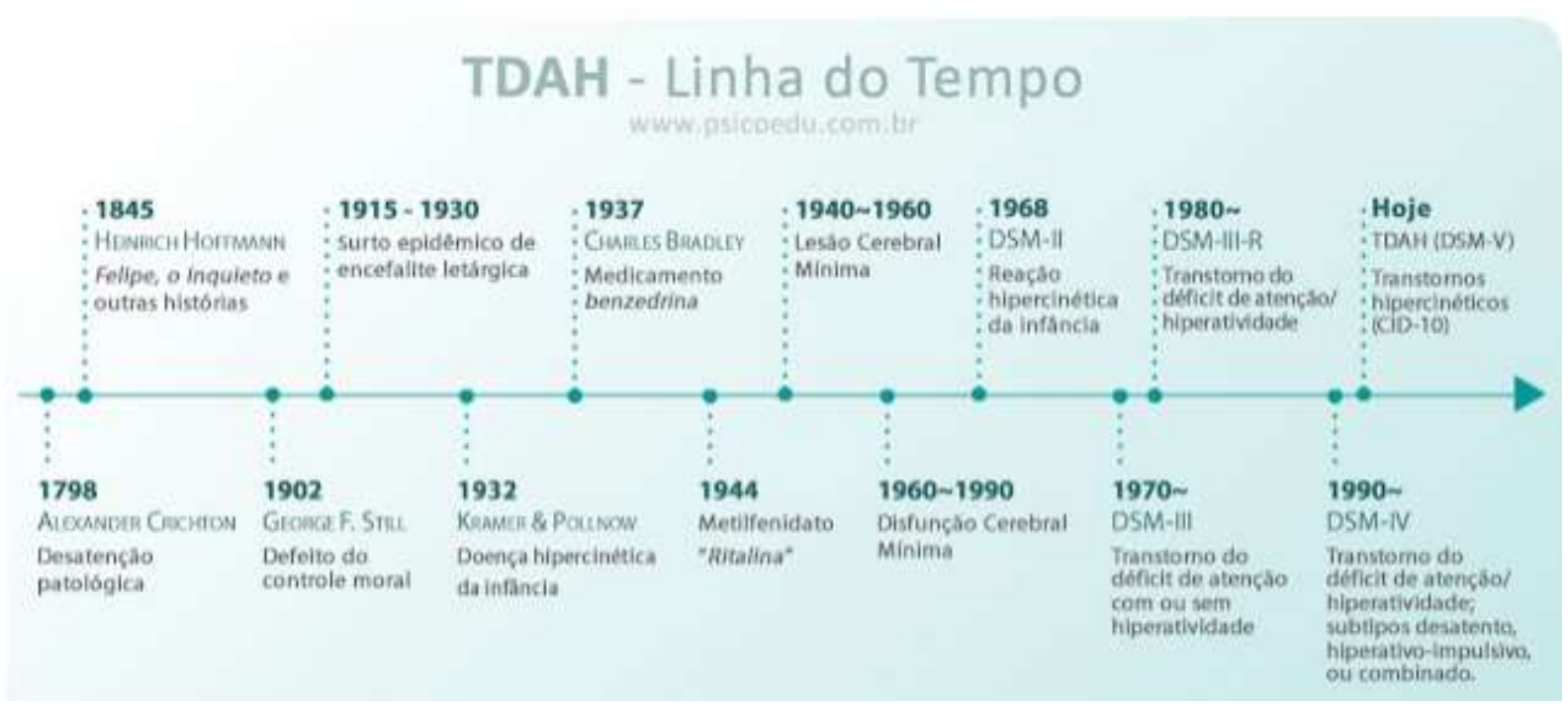

Fonte: Psicoedu (2016).

\section{Algumas Notas}

A análise de Russell A. Burkley (1997 apud Caliman, 2010) representa uma direção neurológica cognitiva e conceitua o TDAH como o resultado de um defeito de inibição e da capacidade de autocontrole, sendo também um defeito da vontade 
assim como déficit da moral. Tanto para Burkley, como para Still e Virgínia Douglas (1997) o foco central das teorias a respeito da análise histórica do TDAH é o vínculo estabelecido entre um defeito neurofisiológico do sistema inibitório, o déficit da moral e da vontade (Caliman, 2010).

Para Caliman (2010), a categoria de diagnósticos onde o TDAH se encontra na atualidade foi construída dentro de dilemas morais, políticos, econômicos e tecnológicos que resgatavam, com importantes alterações, os antigos dilemas patológicos da vontade.

A história oficial do transtorno afirma que o pediatra Still (1902) comenta sobre um defeito de controle moral aparecido no King's College Hospital. Sobre Burkley (1997) a história afirma que esse descreve que o princípio da patologia está no defeito da função inibitória da vontade, que se desdobra de diversas formas interferindo na vontade, na moral e no autocontrole.

Pereira (2009) acredita que a categoria de diagnósticos do TDAH é um produto da negociação inerente ao processo de legitimização de patologias, como no caso do TDAH ocorreu a publicação, em 2002, de um Consenso Internacional para tal fim, onde o primeiro autor foi Russel Burkley e foi assinado por cientistas de diversas nacionalidades a fim também de fazer com que a população acredite na condição médica e biológica do TDAH. Tal documento reafirma a existência de inúmeras controvérsias sobre o transtorno.

\section{Considerações Finais}

O TDAH é um transtorno complexo, multifatorial que possui uma sintomatologia diversificada, cuja história caminha juntamente com seu processo evolutivo de descrição como patologia.

Nesse sentido, percebe-se uma grande variedade de significados atribuídos ao longo do tempo ao transtorno, assim como mudanças nas descrições e concepções da patologia, em todos os aspectos desde o biológico ao social.

O consenso concebido para legitimização auxilia nas avaliações e diagnósticos, porém cabe ressaltar a necessidade de equipes multidisciplinares para que o diagnóstico clínico seja legítimo.

A temática apresentada transborda em possibilidades de temas para futuros trabalhos, como o processo de escolarização e estigmatização dos portadores do transtorno.

\section{Referências}

Amorim, C. (2017). O TDAH tem cura? HTTPS://dda deficitdeatencao.com.br/ artigos/tdah-tem-cura.html

Arruda, M. A. (2006). Levados da Breca - um guia sobre crianças e adolescentes com Transtorno do Déficit de Atenção e Hiperatividade (TDAH). Pdf. https://www.estantevirtual.com.br/livros/marco-a-arruda/levados-da-breca/2178283709.

Azevedo, R. M. D. (2015). O TDAH na perspectiva da inclusão. AVM educacional. https://www.avm.edu.br/docpdf/monografias_public adas/posdistancia/50640.pdf

Brzozoowskil, F. S., \& Sandra, C. A. (2009). Transtorno de déficit de atenção com hiperatividade: classificação e classificados. Physis: Revista de Saúde Coletiva, https://www.scielosp.org/article/physis/2009.v19n4/1165-1187/.

Caliman, L. V. (2010). Notas sobre a história oficial do transtorno do déficit de atenção/hiperatividade TDAH. Psicologia Ciência, 30(1). https://www.scielo.br/j/pcp/a/K7H6cvLr349XXPXWsmsWJQq/?lang=pt>.

Cavalcante, D. (2021). TDAH e os desafios da leitura e escrita - relato de caso. Aprenderedivertido.com. https://docero.com.br/doc/sexx05c.

Costa, C. R., Moreira, J. C. C. \& Júnior, M. O. S. (2015). Estratégias de Ensino e Recursos Pedagógicos para o Ensino de Alunos com TDAH em Aulas de Educação Física. Revista brasileira de educação especial. https://www.scielo.br/j/rbee/a/bv9tRkHHtGWrHqp9KXhS7Bw/?lang=pt.

Couto, T. S., Júnior, M. R. M. \& Gomes, C. R. (2010). Aspectos Neurobiológicos do Transtorno do Déficit de Atenção e Hiperatividade (TDAH): uma revisão. Revista Ciências e Cognição. http://cienciasecognicao.org/revista/index.php/cec/article/view/2021.

Cypel, S. (2003). A Criança com Déficit de Atenção e Hiperatividade: Atualização para pais, professores e profissionais de saúde. (2a ed.), Lemos Editorial.

Dsm-IV. (2003). Associação Americana de Psiquiatria. Manual diagnóstico e estatístico de transtornos mentais - DSM. (4a ed.), Tradução Claudia Dornelles. Artes Médicas. 
Research, Society and Development, v. 11, n. 2, e7611225604, 2022

(CC BY 4.0) | ISSN 2525-3409 | DOI: http://dx.doi.org/10.33448/rsd-v11i2.25604

Domingues, L. (2014). TDAH - Tratamento. blog.saude.gov. http://www.blog.saude.gov.br/34273-tdah-atinge-de-3-a-6-da-populacao-mundial-saiba-maissobre-o-transtorno.html.

Dupin, A. A. S. Q. \& Silva, M. O. (2020). Educação Especial e Legislação brasileira: revisão de literatura. Scientia Vitae. http://revistaifspsr.com/v10n297690.pdf.

Ferreira, R. R., \& Moscheta, M. dos S. (2019). A Multiplicidade do TDAH nas Diferentes Versões Produzidas pelas Ciências no Brasil. Psicologia Escolar e do Desenvolvimento, Psicologia: Teoria e Pesquisa, 35 https://www.scielo.br/j/ptp/a/qwcxTtB4FJSvJMBBtQpqBNK/?lang=pt.

Freire, A. C. C., \& Pondé, M. P. (2005). Estudo piloto da prevalência de TDAH entre crianças escolares na cidade de Salvador, Bahia, Brasil. Arquivos de Neuropsiquiatria, 63(2), 474-478.

Freire, P. (2007). Pedagogia da Autonomia: saberes necessários à prática educativa. (35a ed.), Paz e Terra (Coleção Leitura).

Gil, A. C. (2008). Métodos e técnicas da pesquisa social. (6a ed.), Atlas

Minayo, M. C. de S., et al. (2009). Pesquisa Social: teoria, método e criatividade. (28a ed.), Vozes

Pereira, C. de S. C. Conversas e controvérsias: uma análise da constituição do TDAH no cenário científico e educacional brasileiro. Pdf. Casa de Oswaldo Cruz, 176 f. http://www.ppghcs.coc.fiocruz.br/images/teses/dissertacaoclaricedesa.pdf.

PROIS, Projeto Inclusão Sustentável. TDAH - Transtorno do Déficit de Atenção e Hiperatividade - uma conversa com educadores. Pdf. p 4-33, Brasil, 2021. https://www.psicoedu.com.br/2016/11/historia-origem-do-tdah.html>.

Rezende, E. de. (2021). A história completa do TDAH que você não conhecia. Psicoedu, 